Original paper

\title{
Models of breast lesions based on three-dimensional X-ray breast images
}

\author{
Nikolay Dukov ${ }^{\mathrm{a}}$, Kristina Bliznakova,*, Firgan Feradov ${ }^{\mathrm{a}}$, Ivan Buliev ${ }^{\mathrm{a}}$, Hilde Bosmans ${ }^{\mathrm{b}}$, \\ Giovanni Mettivier ${ }^{\mathrm{c}}$, Paolo Russo ${ }^{\mathrm{c}}$, Lesley Cockmartin ${ }^{\mathrm{b}}$, Zhivko Bliznakov ${ }^{\mathrm{a}}$
}

${ }^{a}$ Laboratory of Computer Simulations in Medicine, Technical University of Varna, Varna, Bulgaria

${ }^{\mathrm{b}}$ Department of Radiology, Katholieke University of Leuven, Leuven, Belgium

' Dipartimento di Fisica "Ettore Pancini", Universita' di Napoli Federico II and INFN Sezione di Napoli, Naples, Italy

\section{A R T I C L E I N F O}

\section{Keywords:}

Breast lesions

Segmentation

Breast tomosynthesis

Dice similarity coefficients

\begin{abstract}
A B S T R A C T
This paper presents a method for creation of computational models of breast lesions with irregular shapes from patient Digital Breast Tomosynthesis (DBT) images or breast cadavers and whole-body Computed Tomography (CT) images. The approach includes six basic steps: (a) normalization of the intensity of the tomographic images; (b) image noise reduction; (c) binarization of the lesion area, (d) application of morphological operations to further decrease the level of artefacts; (e) application of a region growing technique to segment the lesion; and (f) creation of a final 3D lesion model. The algorithm is semi-automatic as the initial selection of the region of the lesion and the seeds for the region growing are done interactively. A software tool, performing all of the required steps, was developed in MATLAB. The method was tested and evaluated by analysing anonymized sets of DBT patient images diagnosed with lesions. Experienced radiologists evaluated the segmentation of the tumours in the slices and the obtained 3D lesion shapes. They concluded for a quite satisfactory delineation of the lesions. In addition, for three DBT cases, a delineation of the tumours was performed independently by the radiologists. In all cases the abnormality volumes segmented by the proposed algorithm were smaller than those outlined by the experts. The calculated Dice similarity coefficients for algorithm-radiologist and radiologist-radiologist showed similar values. Another selected tumour case was introduced into a computational breast model to recursively assess the algorithm. The relative volume difference between the ground-truth tumour volume and the one obtained by applying the algorithm on the synthetic volume from the virtual DBT study is $5 \%$ which demonstrates the satisfactory performance of the proposed segmentation algorithm. The software tool we developed was used to create models of different breast abnormalities, which were then stored in a database for use by researchers working in this field.
\end{abstract}

\section{Introduction}

Breast cancer is by far the most frequently diagnosed cancer and the leading cause of cancer-related death among women worldwide [1]. Despite technological advances, such as the digital mammography, the national screening programs, as well as the introduction of the computer-aided detection (CAD) systems in clinical routine, screening and diagnosis of breast cancers hidden in dense parenchyma still remains a challenging task.

Dedicated CAD systems, including machine learning systems can assist in the detection and classification of the various types of breasts $[2,3]$. Their development and refinement requires a large number of images containing different types of benign and malignant formations. Such images are also useful in the development of new methods for early detection and correct diagnosis of breasts such as digital breast tomosynthesis (DBT) [4], computed tomography dedicated to the breast (BCT) $[5,6]$ and phase-contrast mammography (PCM) $[7,8]$, as well as for the training of young professionals. Obtaining images of real benign and malignant formations of a variety of breasts is however a challenging task, given the limited access to breast cadaver data in cases of breast cancer.

Alternatively, one could analyse images generated by computer simulation of various imaging modalities. Simulated images are obtained using computational models of the breast in which realistic computational models of the breast lesions are incorporated. The computer technology offers wide range of techniques such as constructive solid geometry, voxel (tomographic), polygon-mesh, and hybrid techniques, to model the breast as accurately as possible with all anatomical details, which arise during its development and between different individuals. Anthropomorphic voxel breast phantoms with realistic tissue

\footnotetext{
* Corresponding author.

E-mail address: kristina.bliznakova@tu-varna.bg (K. Bliznakova).
} 
distributions can be also created from clinical data by appropriate digital image segmentation. Patient clinical data may be acquired via breast computed tomography [5,9-12] or magnetic resonance imaging [13-15]. Such an approach guarantees a very high degree of realism (i.e. conformity to real anatomical structures); however, it may represent a single breast composition at a time and lacks the flexibility to cover wide anatomical variations from breast to breast.

Another, hybrid approach (using both solid based and voxel based representations) was proposed by Bliznakova et al. [16,17] and Bakic et al. $[18,19]$ to create a 3D anatomically realistic breast model, which is used to generate synthetic mammograms and tomograms. In the Laboratory of computer simulations in medicine (http://csmlab.tu-varna. $\mathrm{bg}$ ), the research is based on the model of Bliznakova et al. This model was recently evaluated to be suitable for use with cone beam breast CT imaging modality [20]. A modified version of the approach in Bliznakova et al. [16] has been recently adopted by Jeon et al. [21] for generating polychromatic projections in BCT for breast models without abnormalities. The algorithm devised by Bliznakova et al. allows to generate 3D non-compressed breast computer models and some lesions with regular shapes, such as cysts and microcalcifications. Such models can be implemented also physically, with suitable 3D printing materials [22]. The 3D computer model includes a breast-like shape filled with glandular tissue - lactic trees ending with terminal lobular units and Cooper ligaments, lymph and blood systems, pectoral muscle, skin and various benign breast-shaped structures. No irregular lesions have been so far modelled and included in that breast model.

Breast cancer computational models are important for the development of new breast imaging techniques, as well as for realistic models for X-ray breast dosimetry [23,24]. As a large number of different breast cancer models would be normally used, there is a strong need to develop a method for generating patterns of irregular formations, typically in the case of malignant tumours.

One approach is to parameterize the breast lesions. Advanced models of spiculated computational tumour model has been reported by Elangovan et al. $[25,26]$. Their method is based on the extraction of a number of different features from real spiculated tumour masses. These features are used for the generation of digital models of spiculated carcinomas, which are then inserted in real 2D mammography images. As a final step, the generated images are compared with real images of patients with spiculated tumours for evaluation purposes.

Another approach is to use clinical images from modalities that provide 3D breast images, such as DBT and BCT. Such an approach for automatically segmenting breast masses from tomosynthesis images has been reported by several research groups [27-33]. Reiser et al. adopted the radial-gradient index as a feature for mass detection and segmentation in 21 sets of DBT. Peters et al. [34] used active contour models, which evolve a curve in a given image in order to detect objects in that image. The assessment of the algorithm could not be performed directly since there was no ground-truth tumour model. They evaluated the proposed algorithm following three steps: (a) involvement of an expert who outlined contours of masses on mammograms: these contours were then treated as references; (b) applying the algorithm on simulated images, (c) visual assessment of the segmentation results, performed by an expert.

We further extended the above approach to use images from different DBT systems, a method which may be successfully implemented as well as for images from BCT. The main objective of this work was to extract and store voxel-based models of breast lesions with irregular shapes from medical image data from patients. Moreover, we developed algorithms for automatic segmentation of tumours in images from DBT (respectively BCT and CT) which were implemented and evaluated both subjectively and objectively. Successfully segmented 2D slices were then stacked to assemble the 3D shape of the tumour. The created tumour models were stored in a dedicated database. We will continue developing and enriching the tumour model collection, to parameterize the observed shapes and make them available to other interested researchers. As an illustration of the application of the outcomes of this work we provide an example of embedding one of the extracted tumour shapes into an existing healthy breast phantom and using it to simulate realistic breast images.

\section{Materials and methods}

\subsection{Clinical data}

For the purposes of the creation of an algorithm to segment tumour data from 3D patient images, we used 4 out of 50 sets from DBT of patients diagnosed with lesions [35]. The four tomosynthesis sets were acquired with Giotto Tomo, IMS unit (Alexandrovska University Hospital, Sofia, Bulgaria) and with Siemens Mammomat Inspiration (University Hospital of Leuven, Leuven, Belgium). The number of tomosynthesis slices in these sets ranges from 40 to 60 , each reconstructed tomosynthesis slice having a thickness of $1 \mathrm{~mm}$. In the first case IMS unit, tomosynthesis sets are obtained from 13 projections, with a pixel size of $90 \mu \mathrm{m}$, acquired over an arc of 40 degrees, while for the second case the number of mammography projection images with a pixel size of $85 \mu \mathrm{m}$ is 25 , acquired in an arc of 50 degrees. Giotto tomosynthesis images are reconstructed using iterative algorithms, while the Siemens images are reconstructed by using filtered backprojection.

\subsection{Algorithm for identifying the cancerous tissue}

Fig. 1 shows the main stages of the algorithm used in segmenting masses from clinical 3D imaging data.

Clinical or simulated images data are the input resource for the algorithm. After an initial handling, mostly related to organizing the files, anonymizing and planning the next actions, a segmentation of the breast lesions is performed through a specific algorithm. The postprocessing operation is used to correct incorrectly segmented tissue regions. Finally, we stacked the slices with segmented breast lesions into 3D volumetric shapes: the data were then additionally organized and stored in a database as available tumour models. Details, concerning each of the steps, are provided below.

\subsubsection{Pre-processing}

All sets of patient images were anonymized prior to any processing. Further, they were normalized in terms of intensity, in the range of $\left[I_{N_{\min }}, I_{N_{\max }}\right]$ to facilitate the subsequent running of automatic image segmentation algorithms:

$I_{N}=I_{N_{\min }}+\frac{\left(I_{N_{\max }}-I_{N_{\min }}\right)}{\left(I_{\max }-I_{\min }\right)}\left(I-I_{\min }\right)$

where $I_{N}$ is the normalized image and $I_{N_{\max }}$ and $I_{N_{\min }}$ are the new grey maximum and minimum values of the image. A specific case for the range $\left[I_{N_{\min }}, I_{N_{\max }}\right]$ is $[0,1] . I$ is the original image, while $I_{\max }$ and $I_{\min }$ are its maximum and minimum grey values, respectively. In addition, we applied a $3 \times 3$ median filter (taking into account the resolution of the original images) in order to decrease the noise in the original images before the main segmentation process.

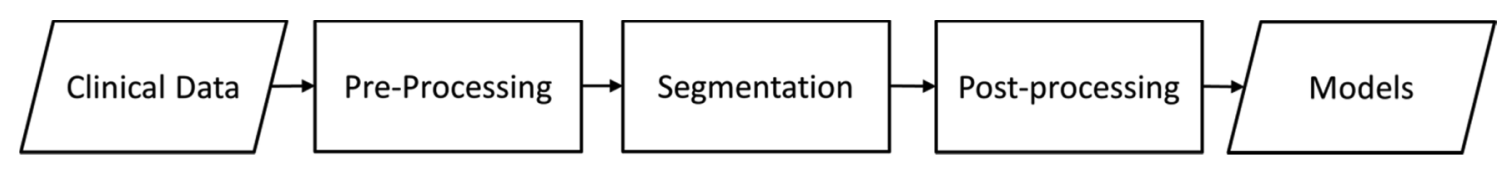

Fig. 1. Main steps of the algorithm for extracting cancerous tissues. 


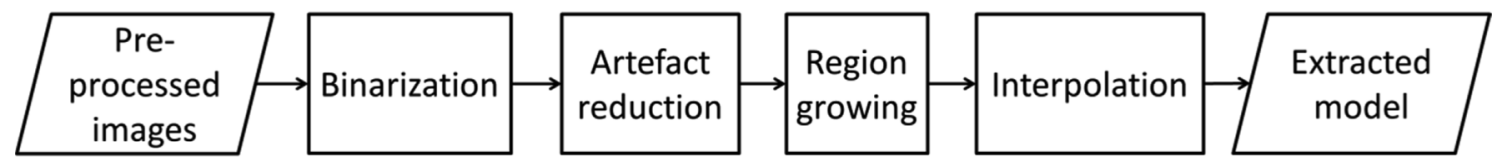

Fig. 2. Main steps in the segmentation algorithm.

\subsubsection{Breast lesion segmentation}

To identify the 3D shapes of the tumours from the available datasets, the proposed approach requires segmentation of the tumours in the tomograms. As this proved to be a largely time-consuming task, efforts were made to develop an automatic segmentation, whose scheme is outlined in Fig. 2.

The input for the algorithm is a set of images, to be processed by the segmentation algorithm, as well as a rough selection in a single image of the tumour region - i.e. by specifying a Region of Interest (ROI). The corresponding ROI for the rest of the images is automatically defined.

2.2.2.1. Binarization. The rough separation between cancerous and healthy tissue is achieved by binarization of the ROI based on an adaptive thresholding.

First, we created a histogram of the unique values in each ROI. The histogram contains 10 equally spaced bins. This number was empirically derived from 20 test patient sets and then validated on another 20 sets of images. Then a lower threshold value $T_{L}$ is set to the intensity corresponding to the bin preceding the bin with the maximum number of elements $\boldsymbol{h}_{\text {max }_{k}}$ (k - the ROI number). The pixels with values under the threshold $T_{L}$ are set to zero.

In the second step, we defined a higher threshold value $T_{H}$. We introduced an empirically defined parameter $\beta$, which is set to 0.7 for DBT, and to 1.0 for images from CT and BCT. The threshold value $T_{H}$ is determined according to:

a) Finding the maximum value $h_{\max }$ :

$h_{\max }=\max _{k=1: N} h_{\max }$

$\mathrm{N}$ - total number of ROI

b) Calculating the threshold value $T_{H}$ :

if $\left(h_{\max }>\beta\right) T_{H}=\frac{1}{N} \sum_{i=1}^{N} h_{\max }, e l s e T_{H}=\beta$

Then, for each ROI the values higher than $T_{H}$ are set to zero:

if $\left(I_{N}(i, j)>T_{H}\right) I_{N}(i, j)=0$

where $(i, j)$ is the pixel coordinates.

Finally, the mean of the new unique values for each ROI is used as a threshold value $T_{d}$ to produce the binary image.

2.2.2.2. Artefact reduction. We observed that, after the binarization of the lesion area, pixels not being part of the tumour formation are often present. These can be single pixels spread around the selected area or artefacts connected to the lesion formation. Morphological operations (erosion, dilation, opening, closing) have been applied to reduce such artefacts. Based on the experience gained from previous studies, after performing multiple tests in various order configurations, we empirically determined the type, the order and the parameters of these operations [36]. In this algorithm, we remove artefacts within 50 pixels (taking into account the image resolution). Both the erosion and the dilation make use of a diamond structure element, whose size is chosen on an image-by-image basis. By applying the artefact reduction step, we achieved the following effects: (i) removal of artefacts, (ii) correction of the defects, which are a result of the tomographic noise, (iii) preservation of the shape of the segmented object.
2.2.2.3. Region growing. We achieved segmentation of the lesion area by applying a region growing algorithm [36] which contributed to the lesion separation by exploiting the connectivity of the pixels in the binarized ROI. The algorithm requires manual input related to the choice of a starting point - mouse clicks inside the lesion area on an automatically presented binary image of the slice. The coordinates are further automatically transferred to the next slices. The selection was performed on a single slice, which brings convenience to the physician. To prevent potential interruptions, three different starting points need to be selected. Finally, we applied a set of morphological operations to remove any defects (dark pixels inside the tumour shape, rough edges), introduced by the processing.

2.2.2.4. D. Interpolation. The segmentation algorithm performs also validation of integrity across the slices, and includes a comparison of both tumour area size and shape in two adjacent slices. A combination of the areas in these two adjacent slices is considered, whenever a difference is found. The algorithm also takes into account the similarity between the current slice and the two adjacent slices in each direction.

Illustration of the described steps from A to D is given in Fig. 3.

Manual outlining of the tumour regions in the tomographic images was used as a reference during the development of the segmentation algorithm.

\subsubsection{Post-processing (manual corrections)}

An additional outcome from the present work is the development of a software tool with a graphical user interface (GUI). Since the described segmentation procedure and visualisation scripts were developed in Matlab (MathWorks, Inc.), the GUI (Fig. 4) was naturally developed to facilitate the user work.

This tool permits to browse the original and the segmented tumour images and to perform a subjective evaluation and corrections. The user is given the opportunity to add or delete parts of the segmented regions, if needed. Such cases may be when the actual tumour area is less or more than the segmented by the algorithm. In this case under the supervision of the radiologist the segmented area may be corrected.

\subsection{Evaluation and testing}

We applied two types of assessment: $i$ ) by comparing the segmented tumour masses after automatic tumour segmentation to the one outlined by expert radiologists; (b) comparing a ground-truth tumour volume with the one obtained after applying the segmentation algorithm on a synthetic volume, obtained from virtual X-ray tomosynthesis study with a computational breast with this ground-truth tumour model introduced.

Three radiologists with an expertise of more than 15 years in the field of mammography participated in the first assessment type. They were from three different hospitals and from two different EU countries. Three cases of irregular breast cancers were stored in a notebook, supplied with the RadiAnt DICOM Viewer application. The radiologists had spent about $30 \mathrm{~min}$ per case to outline the cancer area, for each slice. They were not instructed to outline the abnormalities following closely the abnormality boundaries. They performed the segmentation without the presence of the team members, and then sent their outlined images. The segmentations performed by the radiologists and the corresponding results of the algorithm were compared with the help of five metrics, presented below. In all expressions, A is the area segmented by the algorithm, while $\mathrm{B}$ is the area outlined by the radiologist. The 

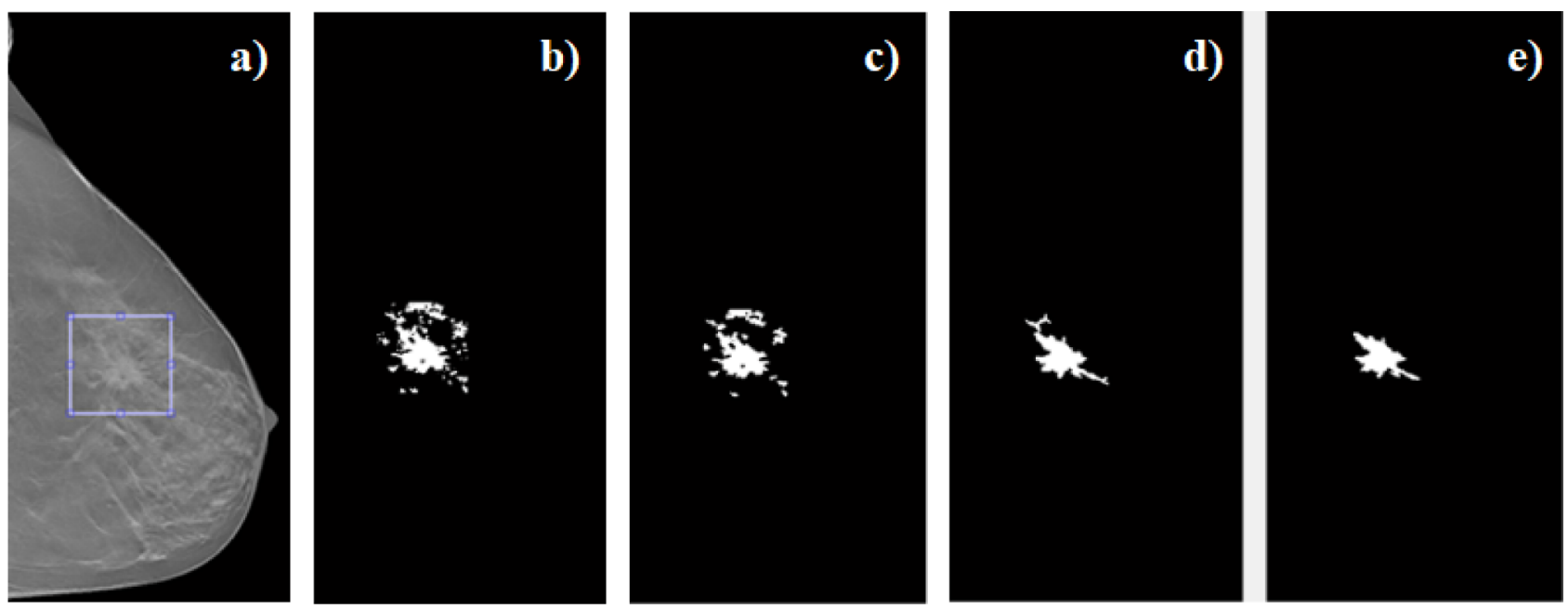

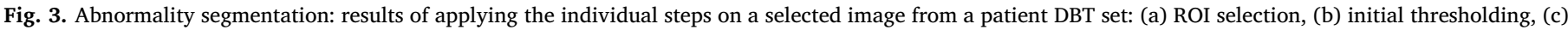
morphological operations, (d) region growing and (e) interpolation.

following metrics were evaluated:

- Dice similarity coefficient (DSC), defined as the intersection of two masks normalized to their mean volume, where A and B are the masks and $\mathrm{N}$ is an operator yielding the number of voxels [37]:

$D S C=\frac{2 * N(A \cap B)}{N(A)+N(B)}$

DSC range is $[0,1]$ where zero indicates no overlap and one indicates exact overlap.

- Percentage relative volume difference (RVD) in percentage, defined as:

$R V D=\frac{|A-B|}{B} * 100$

A value of 0 indicates that both volumes are identical.

- VOSA (VOlume Selected by the Algorithm) - a metric that estimates the volume selected by the algorithm but not selected by the radiologist as percentage of the volume selected by any of them:
$\operatorname{VOSA}=\frac{|A \cup B-B|}{A \cup B} * 100$

If the volume selected by the algorithm is entirely included in the one selected by the physician, then VOSA equals 0 . This is illustrated in Fig. 5a for a single tomographic slice, where two such regions are present (denoted as $C$ ).

- VOSR (VOlume Selected by the Radiologist) - a metric that estimates the volume selected by the radiologist but not selected by the algorithm as percentage of the volume selected by any of them:

$\operatorname{VOSR}=\frac{|A \cup B-A|}{A \cup B} * 100$

In this case, if the volume selected by the radiologist is within the one selected by the algorithm, then VOSR equals 0 . This is illustrated in Fig. 5b, where two such regions are present (denoted as $C$ ).

For the second assessment type, the proposed method was applied to tomosynthesis slices obtained via simulations of a ground-truth breast volume. This ground-truth model was created from a fourth patient DBT data set by applying the algorithm. The voxelised tumour

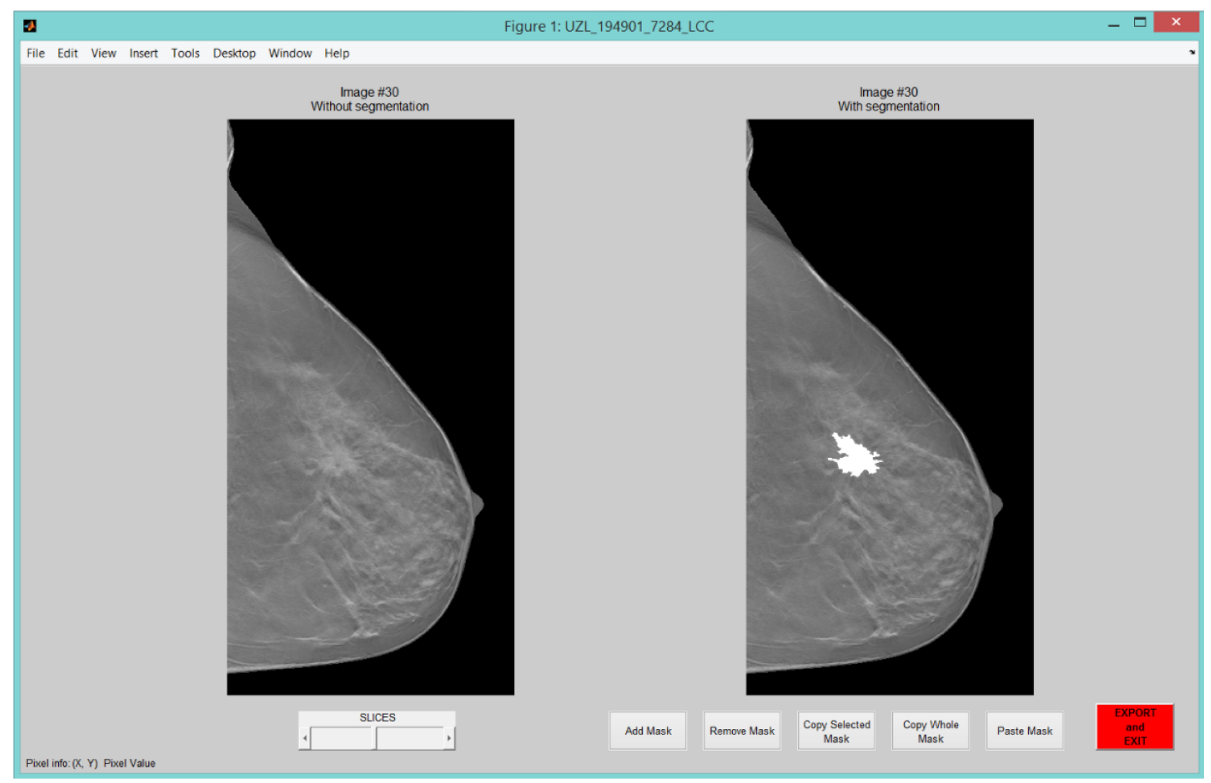

Fig. 4. A screenshot of the GUI dedicated to the tumour segmentation and further processing. 


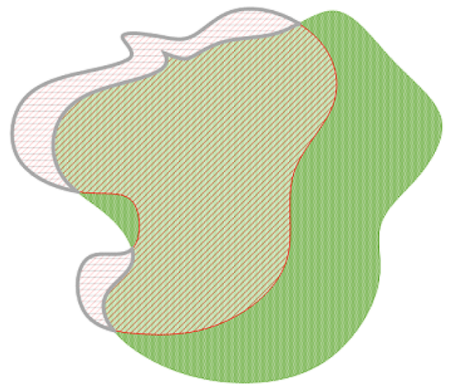

a)

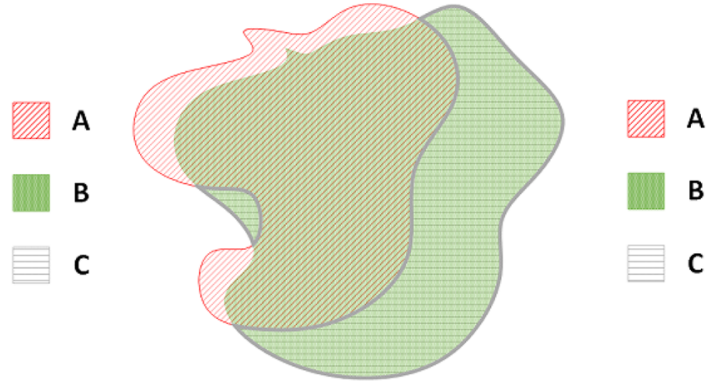

b)

Fig. 5. The result for VOSA (a) $A \cup B-B$ and (b) VOSR $A \cup B-A$.

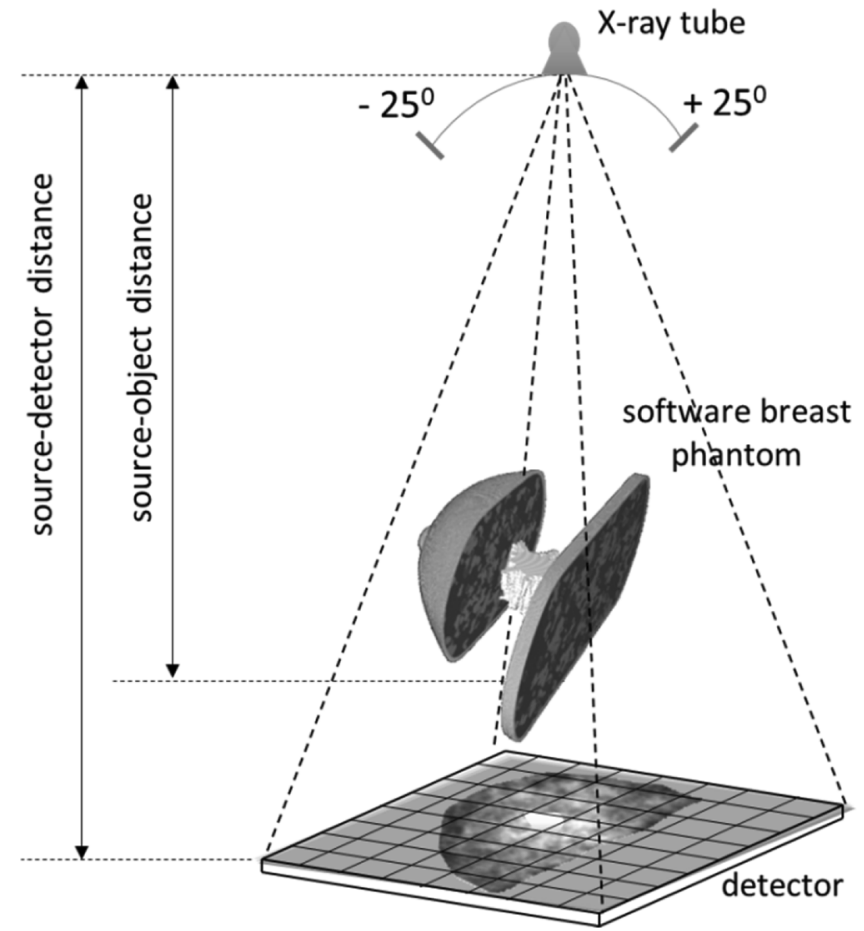

Fig. 6. Simulated tomosynthesis imaging: the generated breast phantom with the integrated abnormality and the acquisition arc.

matrix was $414 \times 455 \times 423$ voxels, with each voxel sized $0.2 \mathrm{~mm}$. This study included the insertion of the 3D segmented lesion into an anthropomorphic breast phantom mimicking a compressed dense female breast with a thickness of $40 \mathrm{~mm}$, created with a dedicated BreastSimulator software application [38]. The cubic voxel size of the breast phantom was $0.2 \mathrm{~mm}$ in each direction. The acquisition setup is indicated in Fig. 6. It includes the generation of 26 noise-free X-ray projection images (from $-25^{\circ}$ to $25^{\circ}$ with a discrete step of $2^{\circ}$ ) free of noise from the breast phantom with the ground-truth tumour model by simulating the penetration of a $20 \mathrm{keV}$ monochromatic X-ray beam. The distances from the source to the breast support table, where the phantom is placed, and to the detector surface, were $600 \mathrm{~mm}$ and $650 \mathrm{~mm}$, respectively. The size of the images is $1200 \times 1200$ pixels, and each pixel with dimensions of $0.085 \mathrm{~mm} \times 0.085 \mathrm{~mm}$.

The 26 projection images were then used with an in-house developed reconstruction tool [39] to obtain tomosynthesis images, so building the 3D reconstructed breast volume. Further, the proposed algorithm was tested on this volume and the segmented tumour was compared to the ground-true (originally segmented) cancer volume.

\section{Results and discussion}

Fig. 7 depicts an example of applying the segmentation method to DBT data of a 56-year-old female with a unifocal moderately differentiated invasive ductal adenocarcinoma (IDA); this $2.5-\mathrm{cm}$ breast cancer was classified as BIRADS 5. The indications on the mammogram were stellate distortion retro areolar.

One can observe the small contrast difference between the tumour and the neighbouring regions. The thickness of the reconstructed tomosynthesis slices is $1 \mathrm{~mm}$; the segmented lesion is shown in Fig. $7 \mathrm{~d}$. Further image processing may be applied to smooth the final segmented lesion.

\subsection{Evaluation by radiologists}

Fig. 8 shows a comparison of segmented slices obtained by using the algorithm (Fig. 8b, first row) and outlined by the three radiologists (Fig. 8c-e, first row) and the corresponding volumes (Fig. 8b-e, second row). The $3 \mathrm{D}$ images of the segmented tumours are shown under the
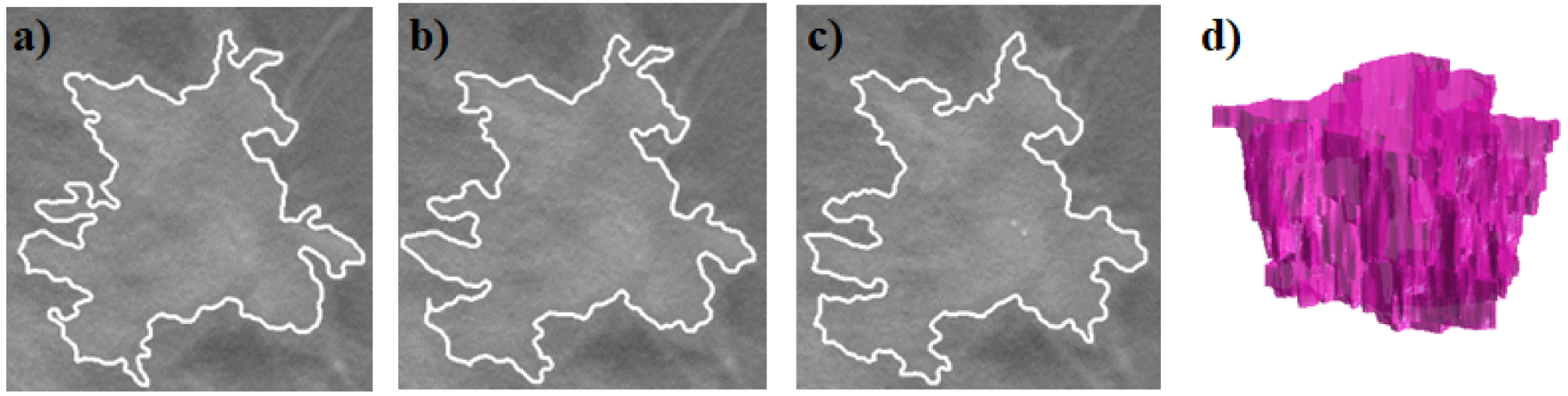

Fig. 7. An example of the application of the proposed method to segment the abnormality: (a-c) three sequent segmented slices, (d) the visualization of the segmented lesion. 


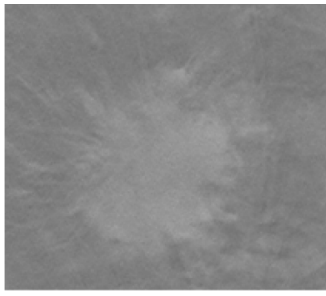

a)
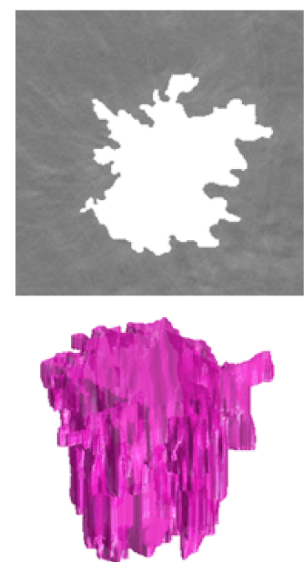

b)
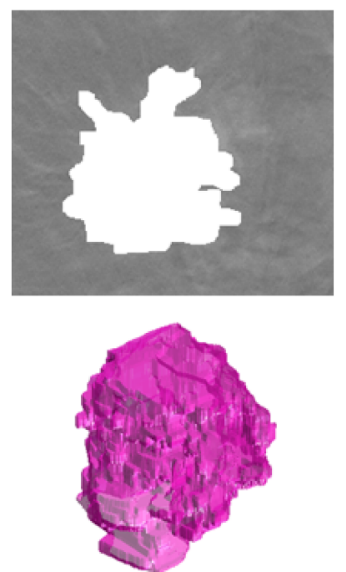

c)
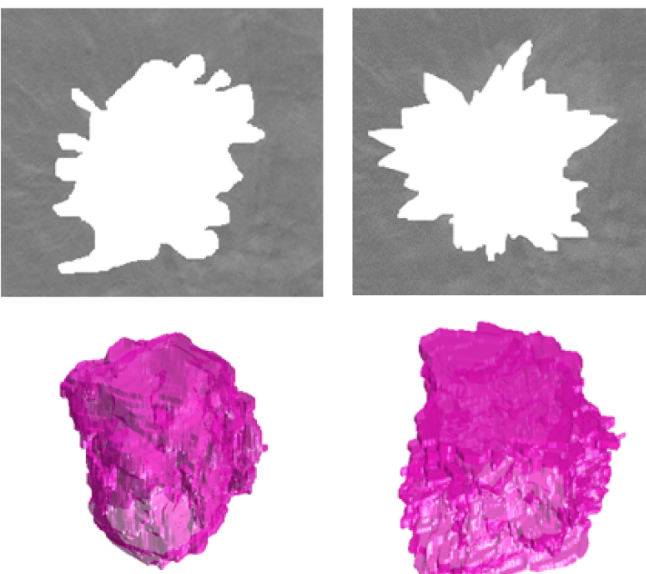

d)

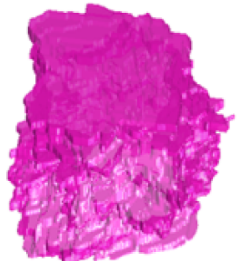

e)

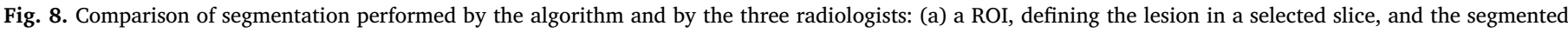

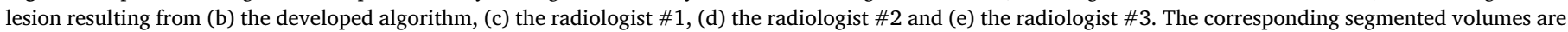
shown in the second row.

corresponding slices.

The visual comparison shows that the area segmented by the algorithm covers the cancer area as the one segmented by the three radiologists. The area segmented by the algorithm was smaller and too irregular compared to the area, segmented by the radiologists. This is expected for several reasons: (a) the specific breast tumour is very irregular; (b) the algorithm segments at a level of detail limited by the image resolution and the characteristics of the applied filters; a segmentation by hand would be coarser; (c) the radiologists in general were not interested in drawing the exact tumour borders, rather they preferred to outline a broader area, so as to be absolutely sure that the cancer is contained in the selected area.

This is well demonstrated in Fig. 9, where a detailed comparison of outlined tumour's contour by the three radiologists and the algorithm is shown for a randomly chosen patient and slice. It can be observed that the area segmented by the algorithm is always smaller than the area segmented by the radiologists. It is also visually notable the different performance by the three radiologists for the same patient case.

In order to have similar results to the ones of the radiologists, image processing operations such as dilation may be applied to expand the area segmented by the algorithm and to smooth the contour as well. However, this is not a goal of this study, while this function may be added as new functionality.

Table 1 summarises the results for three patient cases (P1, P2 and P3) of the objective evaluation based on Dice similarity coefficients. The DSCs ranges between 0.56 and 0.76 , which supports the conclusion of other studies [37] that radiologists' performance differs largely among them, so the accuracy of so-derived ground-truth/gold standard reference models for evaluation of computer segmentation algorithms can be questioned. This is also shown in Table 2 for DSCs calculated for radiologist vs radiologist test. This coefficient ranges between 0.39 and 0.85 , indeed a wider range than the one calculated for algorithm vs radiologist performance. The case of DSC 0.39 is illustrated in Fig. 10 for two slices where a large difference is observed between radiologists' delineations. However, the aim of this work is to produce such threedimensional tumour models, the requirement for which is to look realistic in shape and form, rather than to be exact copy of the real tumour, which is also difficult to segment with very high accuracy and evaluate correctly. Therefore, the feasibility of applying this methodology is shown on three patient DBT data sets, while segmented tumour data and their arrangement is presented in [35]. We are currently organizing a large study on the set of images in the database to investigate the discrepancy between radiologists for this specific case of tomosynthesis, where images are of low contrast.
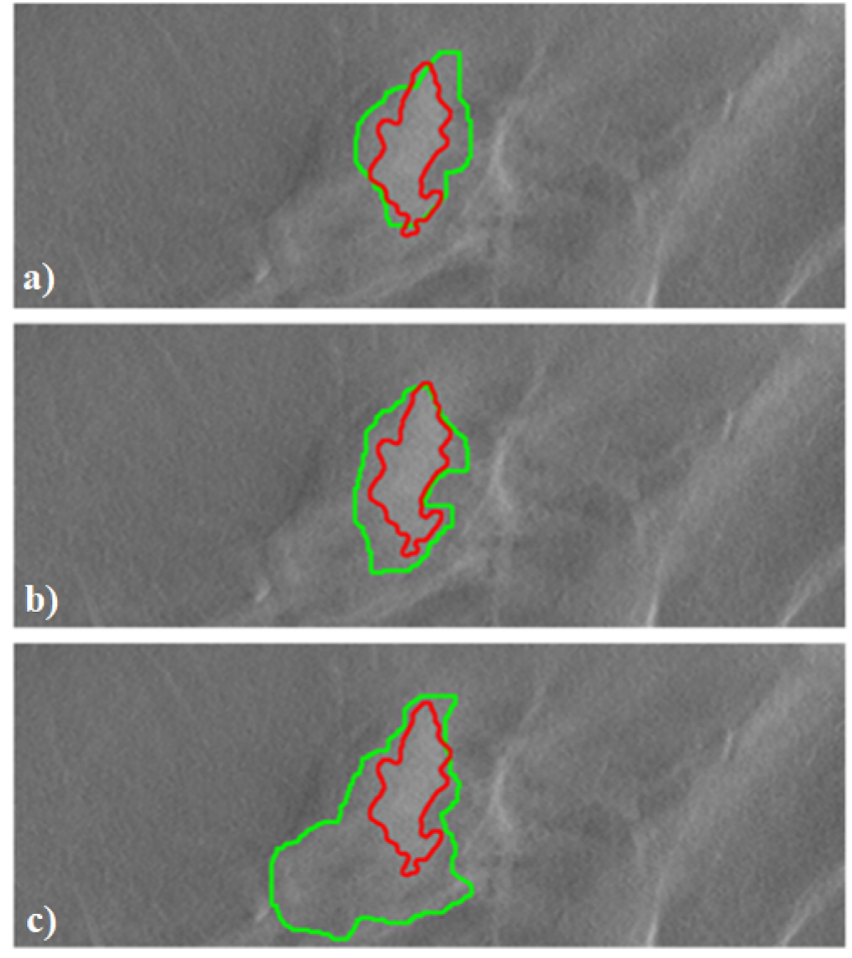

Fig. 9. A comparison of segmented (on tomographic slices) shapes obtained with the algorithm (in red) and the three radiologists (green) a-c who participated in the study.

Table 1

Dice similarity coefficients for algorithm-radiologist and radiologist-radiologist.

\begin{tabular}{|c|c|c|c|c|c|c|c|}
\hline & \multicolumn{3}{|c|}{ DSC $($ Full match $=1)$} & \multicolumn{3}{|c|}{ DSC $($ Full match $=1)$} & \\
\hline & $\mathrm{P} 1$ & P2 & P3 & $\mathrm{P} 1$ & $\mathrm{P} 2$ & P3 & \\
\hline A-R1 ${ }^{*}$ & 0.74 & 0.69 & 0.76 & 0.85 & 0.80 & 0.60 & R1-R2 ${ }^{* * *}$ \\
\hline A-R2 & 0.72 & 0.74 & 0.65 & 0.84 & 0.72 & 0.62 & R1-R3 ${ }^{* * k}$ \\
\hline A-R3 & 0.71 & 0.59 & 0.56 & 0.85 & 0.73 & 0.39 & R2-R3 ${ }^{* * *}$ \\
\hline
\end{tabular}

* A-R1, A-R2, A-R3-algorithm vs radiologist performance.

** R1-R2, R1-R3, R2-R3-radiologist vs radiologist performance. 
Table 2

Calculated metrics between volumes segmented by the algorithm and radiologists.

\begin{tabular}{|c|c|c|c|c|c|c|c|c|c|}
\hline & \multicolumn{3}{|c|}{ RVD (Full match $=0$ ) } & \multicolumn{3}{|c|}{ VOSA (Full match $=0$ ) } & \multicolumn{3}{|c|}{ VOSR (Full match $=0$ ) } \\
\hline & $\mathrm{P} 1$ & $\mathrm{P} 2$ & P3 & $\mathrm{P} 1$ & $\mathrm{P} 2$ & P3 & $\mathrm{P} 1$ & $\mathrm{P} 2$ & P3 \\
\hline A-R1 ${ }^{*}$ & 29.66 & 50.05 & 23.28 & 6.03 & 4.04 & 12.67 & 34.96 & 43.77 & 26.14 \\
\hline A-R2 & 39.87 & 43.85 & 20.72 & 0.49 & 4.63 & 45.57 & 43.31 & 36.89 & 6.71 \\
\hline A-R3 & 43.67 & 62.93 & 63.00 & 2.47 & 2.56 & 2.99 & 42.07 & 56.04 & 58.62 \\
\hline
\end{tabular}

* A-R1, A-R2, A-R3 - algorithm vs radiologist performance.

Further analysis for the rest of the metrics, summarized in Table 2, shows that RVD is always greater than $0 \%$, which indicates that the volume of the segmented abnormality is always less than the one segmented by the radiologists, as expected. In general, the VOSA values are small, a circumstance in line with the observation in Fig. 9 that the regions outlined by the algorithm outside the area outlined by the radiologists, have relatively very small area. The values for VOSR are also expected to show that the volumes selected by the three radiologists differ from the one selected by the algorithm in the range between $7 \%$ and $59 \%$, which is well seen in the comparison demonstrated in Fig. 9, for all three radiologists.

This study did not include the use of STAPLE algorithm [40]. The scope of an ongoing MSc thesis study originating from this work, is to create a ground-truth model from clinical delineations and carefully evaluate the algorithm. We are undertaking several steps to improve the application of this method. One is related to the software for delineation, which needs to be better suited for the radiologists' work: we are currently developing improved interactive tools in order to facilitate the radiologists in delineating the cases. Another step forward is related to the development of a dedicated protocol for addressing the main points during delineation process: initial training with the images and accurate delineation of abnormalities, with the addition of new cases in the database.

\subsection{Evaluation of the algorithm against a ground-truth tumour volume}

Fig. 11a shows slices and tumour volume segmented by the algorithm from patient images. The next three columns in Fig. 11 show the corresponding slices segmented by the same algorithm from simulated images of a breast phantom, in which the previously segmented tumour was incorporated.

A good visual coincidence between the two datasets is evident. We calculated a relative difference of $5 \%$ between the ground-truth tumour volume and the one obtained by applying the algorithm on the reconstructed volume from the virtual DBT. In case of simulated tomosynthesis, the segmented abnormality was surrounded by a dense tissue, which made the segmentation a challenging task. This also
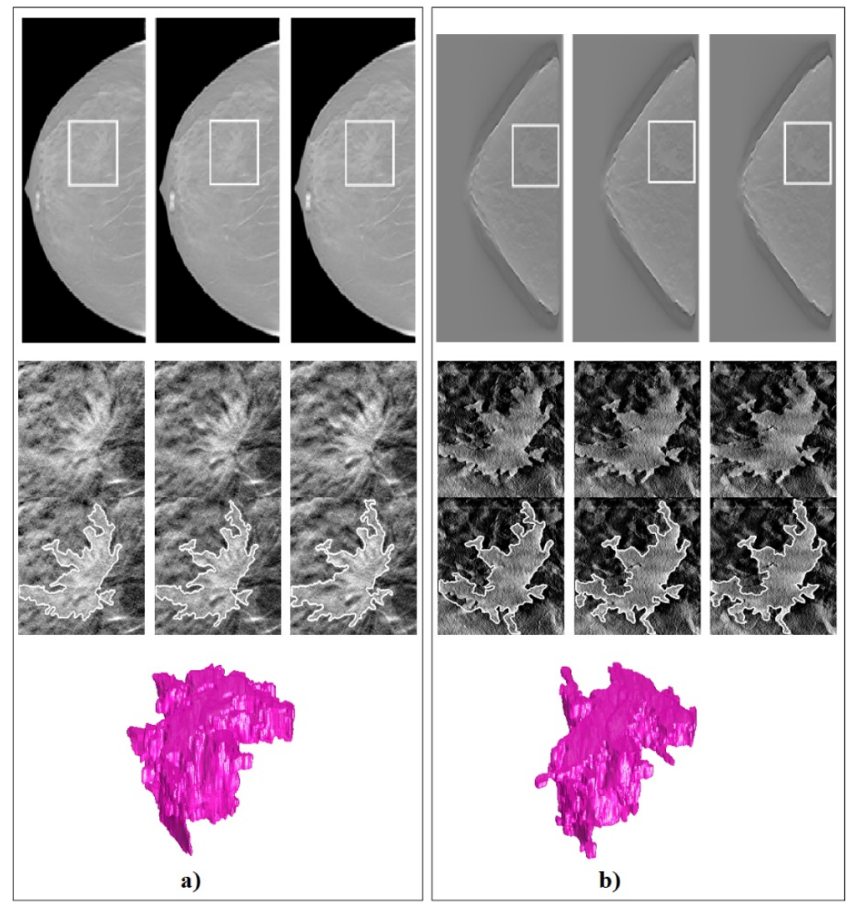

Fig. 11. Recursive application of the algorithm: (a) segmentation from real tomosynthesis and (b) segmentation from simulated tomosynthesis.

implies that the use of these models may well fit the needs of virtual studies aiming at developing and optimising present and new X-ray breast imaging technologies as well as CAD algorithms.

\section{3. $3 D$ tumour shapes and database}

Segmented 3D volumes containing the segmented shape and relevant information about it were created and saved in a separate

\section{first patient}

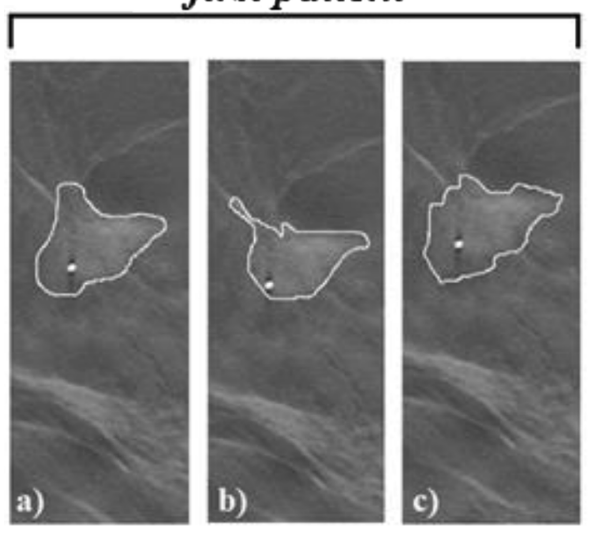

second patient

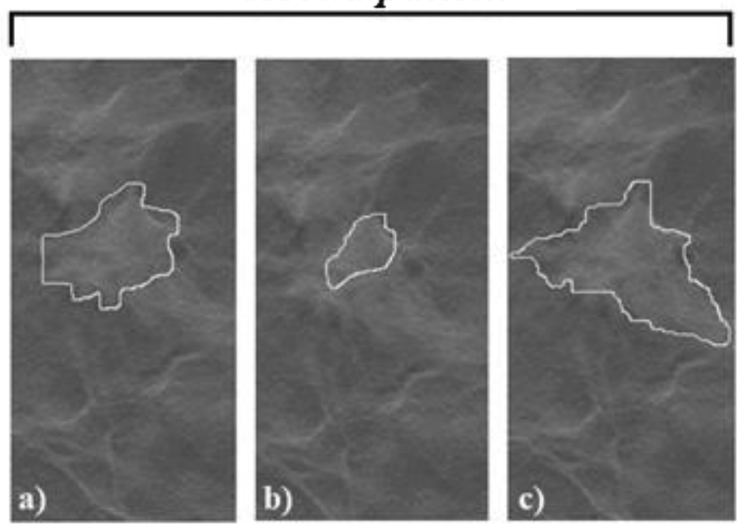

Fig. 10. Comparison of radiologists' performance for two patient cases, one slice per patient: (a) radiologist 1, (b) radiologist 2, (c) radiologist 3. 
MATLAB data file. To facilitate the further processing, analysis and computations, two types of files are saved. The first one contains the segmented abnormality (in grey values) and the necessary information related to the file structure named info structure (available also as a text file). The second file type contains two sets of images - the original ROI slices and the binary images of the segmented tumour shape, as well as a description related to these file structures. The file structure always contains the tumour model's name, the volume and voxel dimensions, the geometrical centre of the tumour model, displayed range and the original pixel values.

The segmented tumour images were then stacked by considering the aspect ratio of the pixels in the tomographic images and the spatial distribution of tomographic slices. Further, they are placed in a database repository presented in [35]. The tumour shapes are also saved as raw data in binary file, accompanied with general information, which should allow for data handling.

\section{Conclusions}

This work presents an approach to obtain voxel-based models of breast lesions with irregular shapes from patient breast tomosynthesis data. The proposed algorithm will facilitate the processing of any new set of DBT and BCT images, as well as images from breast cadavers and whole body CT and speed up the development and population of database with various tumour models. Collecting and analysing a sufficiently large number of cancer shapes will also serve as a basis for developing a mathematical framework for automatic generation of computational breast cancer models. The models can be then used in advanced virtual X-ray breast imaging studies, e.g. phase contrast breast tomosynthesis.

\section{Acknowledgments}

This work is supported by MaXIMA project, which has received funding from the European Union's Horizon 2020 research and innovation programme under grant agreement No 692097. This work is also supported by the Bulgarian National Science Fund under grant agreement DN17/2.

\section{References}

[1] DeSantis CE, et al. International variation in female breast cancer incidence and mortality rates. Cancer Epidemiol Biomarkers Prev 2015;24(10):1495-506.

[2] Atlas NE, Arouss ME, Wahbi M. Computer-Aided Breast Cancer Detection Using Mammograms: A Review. in Second World Conference on Complex Systems (Wccs); 2014: IEEE.

[3] Liang C, et al. A computer-aided diagnosis scheme of breast lesion classification using GLGLM and shape features: combined-view and multi-classifiers. Phys Med 2018;55:61-72.

[4] Sechopoulos I. A review of breast tomosynthesis. Part I. The image acquisition process. Med Phys 2013;40(1). 014301.

[5] Sarno A, Mettivier G, Russo P. Dedicated breast computed tomography: Basic aspects. Med Phys 2015;42(6):2786-804.

[6] Longo R, et al. Towards breast tomography with synchrotron radiation at Elettra: first images. Phys Med Biol 2016;61(4):1634-49.

[7] Honda C, Ohara H. Advantages of magnification in digital phase-contrast mammography using a practical X-ray tube. Eur J Radiol 2008;68(3 Suppl):S69-72.

[8] Castelli E, et al. Mammography with synchrotron radiation: first clinical experience with phase-detection technique. Radiology 2011;259(3):684-94.

[9] Li CM, et al. Methodology for generating a 3D computerized breast phantom from empirical data. Med Phys 2009;36(7):3122-31.

[10] Huang SY, et al. The characterization of breast anatomical metrics using dedicated breast CT. Med Phys 2011;38(4):2180-91.

[11] Yang X, Sechopoulos I, Fei B. Automatic tissue classification for high-resolution breast CT images based on bilateral filtering. Med Imag 2012 Image Process
2011;7962:79623H

[12] Sarno A, et al. Imaging performance of phase-contrast breast computed tomography with synchrotron radiation and a CdTe photon-counting detector. Phys Med 2016;32(5):681-90.

[13] Pathmanathan P, et al. Predicting tumor location by modeling the deformation of the breast. IEEE Trans Biomed Eng 2008;55(10):2471-80.

[14] Shih TC, et al. Computational simulation of breast compression based on segmented breast and fibroglandular tissues on magnetic resonance images. Phys Med Biol 2010;55(14):4153-68

[15] Stewart ML, Smith LM, Hall N. A numerical investigation of breast compression: a computer-aided design approach for prescribing boundary conditions. IEEE Trans Biomed Eng 2011;58(10):2876-84

[16] Bliznakova K, et al. A three-dimensional breast software phantom for mammography simulation. Phys Med Biol 2003;48(22):3699-719.

[17] Bliznakova K, et al. Evaluation of an improved algorithm for producing realistic 3D breast software phantoms: application for mammography. Med Phys 2010;37(11):5604-17.

[18] Bakic PR, et al. Mammogram synthesis using a 3D simulation. II. Evaluation of synthetic mammogram texture. Med Phys 2002;29(9):2140-51.

[19] Bakic PR, Zhang C, Maidment AD. Development and characterization of an anthropomorphic breast software phantom based upon region-growing algorithm. Med Phys 2011;38(6):3165-76.

[20] Mettivier G, et al. Evaluation of the BreastSimulator software platform for breast tomography. Phys Med Biol 2017;62(16):6446-66.

[21] Jeon H, et al. Generation of polychromatic projection for dedicated breast computed tomography simulation using anthropomorphic numerical phantom. PLoS ONE 2017;12(11). e0187242.

[22] Ivanov D, et al. Suitability of low density materials for 3D printing of physical breast phantoms. Phys Med Biol 2018.

[23] Sarno A, et al. Homogeneous vs. patient specific breast models for Monte Carlo evaluation of mean glandular dose in mammography. Phys Med 2018;51:56-63.

[24] Sarno A, et al. Monte Carlo evaluation of glandular dose in cone-beam X-ray computed tomography dedicated to the breast: Homogeneous and heterogeneous breast models. Phys Med 2018;51:99-107.

[25] Elangovan, P., et al. Simulation of spiculated breast lesions. in SPIE Medical Imaging; 2016: SPIE.

[26] Elangovan P, et al. Design and validation of realistic breast models for use in multiple alternative forced choice virtual clinical trials. Phys Med Biol 2017;62(7):2778-94.

[27] Reiser I, et al. Computerized detection of mass lesions in digital breast tomosynthesis images using two- and three dimensional radial gradient index segmentation. Technol Cancer Res Treat 2004;3(5):437-41.

[28] Peters, G., et al. A hybrid active contour model for mass detection in digital breast tomosynthesis. in Medical Imaging; 2007: SPIE

[29] Singh S, Tourassi GD, Lo JY. Effect of similarity metrics and ROI sizes in featureless computer aided detection of breast masses in tomosynthesis. Springer, Berlin Heidelberg Heidelberg: Berlin; 2008.

[30] Zhang Y, et al. Investigation of different PV distributions in digital breast tomosynthesis (DBT) mammography. Berlin, Heidelberg: Springer Berlin Heidelberg; 2008.

[31] Wei J, et al. Computer-aided detection of breast masses: four-view strategy for screening mammography. Med Phys 2011;38(4):1867-76.

[32] Fotin, S.V., et al. Detection of soft tissue densities from digital breast tomosynthesis: comparison of conventional and deep learning approaches. in SPIE Medical Imaging; 2016: SPIE.

[33] Samala RK, et al. Mass detection in digital breast tomosynthesis: deep convolutional neural network with transfer learning from mammography. Med Phys 2016;43(12):6654.

[34] Peters, G., et al. A Hybrid Active Contour Model for Mass Detection in Digital Breast Tomosynthesis. in Proceedings of SPIE - The International Society for Optical Engineering; 2007: SPIE Medical Imaging.

[35] Bliznakova K, et al. Development of breast tumours models database. Physica Medica 2018. (submitted as an invitated article to the Focus Issue ECMP 2018).

[36] Bliznakova K, et al. Computer aided preoperative evaluation of the residual liver volume using computed tomography images. J Digit Imaging 2015;28(2):231-9.

[37] Deeley MA, et al. Comparison of manual and automatic segmentation methods for brain structures in the presence of space-occupying lesions: a multi-expert study. Phys Med Biol 2011;56(14):4557-77.

[38] Bliznakova $\mathrm{K}$, et al. BreastSimulator: a software platform for breast x-ray imaging research. J Biomed Graph Comput 2012;2(1):1-14.

[39] Kamarianakis Z, Buliev I, Pallikarakis N. A platform for Image reconstruction in Xray Imaging: medical applications using CBCT and DTS algorithms. Comput Sci J Moldova 2014;22(2):236-52.

[40] Warfield SK, Zou KH, Wells WM. Simultaneous truth and performance level estimation (STAPLE): an algorithm for the validation of image segmentation. IEEE Trans Med Imag 2004;23(7):903-21. 